\title{
Analysis of Sheet Fracture Failure Based on XFEM
}

\author{
Xinya Chen ${ }^{1}$, Zhen Chen ${ }^{*}, 1$ and Yang Zhao ${ }^{2}$ \\ ${ }^{I}$ Department of Electronic and Communication Engineering, Henan Mechanical \& Electrical Engineering College, \\ Xinxiang 453003, China \\ ${ }^{2}$ Department of Electronic and Information Technology, Jiangmen Polytechnic, Jiangmen 529090, China
}

\begin{abstract}
Extended finite element method (XFEM) is the most effective numerical method to solve discrete mechanical problem. Crack growth problem of two-dimension finite length rectangle panel is researched based on Abaqus XFEM frame. Stress intensity factor is obtained respectively by theoretical calculation and XFEM simulation, which proves reliability of XFEM and the software.
\end{abstract}

Keywords: XFEM, Fracture mechanics, Stress intensity factor, Static crack simulation.

\section{INTRODUCTION}

Fatigue and fracture problems have long been concerned in solid mechanics and science of material strength. In sheets, shells and other thin-walled structures especially, material defect and stress concentration can usually trigger structural fracture failure. Griffith [1] first proposed the extension condition of crack instability from the perspective of energy balance. Subsequently, Irwin put forward the concept of plastic dissipated power at the crack tip area [2], as well as the further concept of stress intensity factor [3]. In 1968, Rice and other scholars proposed the path-independent J-integral [4]. That same year, Hutchinson et al. established the famous HRR singularity field, which laid an important theoretical framework for elastic-plastic fracture mechanics $[5,6]$. However, later elaborated numerical computation revealed $[7,8]$ that the stress-strain field at crack tip could hardly be represented by the HRR field. Considering such a situation, Li Yaochen and Wang Ziqiang [9] established the basic equations for the high-order field of elastoplasticity at crack tip, and produced the second-order field of plane strain, in 1986. The extended finite element method was proposed by Belytschko and Black [10] to reflect the existence of cracks in the form of additional function while solving crack problems. Moes has modified this method and named it XFEM [11]. Fang Xiujun et al. have implemented the functions of XFEM at software merchants using the method of presupposed joints, with Abaqus as the platform [12]. Based on the basic theory of extended finite element, this paper begins with a description of the stress field at crack tip through numerical computation, and then performs a simulation through finite element software to get separate stress intensity factors.

*Address correspondence to this author at the Department of Electronic and Communication Engineering, Henan Mechanical \& Electrical Engineering College, Xinxiang 453003, China; E-mail: chenzhen22@163.com

\section{BASIC THEORY OF EXTENDED FINITE ELEMENT}

\subsection{Description of Extended Finite Element}

Partition of unity method (PUM) is defined in accord with moving least square method (MLSM). For any function $u(x)$, the partial approximation function at point $x$ is defined as:

$u^{h}(\mathrm{x})=\sum_{i=}^{r} p_{i}(\mathrm{x}) \mathrm{a}_{\mathrm{i}}(\mathrm{x})=p^{T}(\mathrm{x}) \mathrm{a}(\mathrm{x})$

where, $p_{i}(\mathrm{x})$ is the primary function, $a_{i}(\mathrm{x})$ is the corresponding coefficient.

$J(\mathrm{a}(\mathrm{x}))=\sum_{i=1}^{r} \phi_{i}(\mathrm{x})\left[\mathrm{p}^{\mathrm{T}}\left(\mathrm{x}_{\mathrm{i}}\right) \mathrm{a}(\mathrm{x})-\mathrm{u}_{\mathrm{i}}\right]^{2}$

where, $x_{i}$ is the node of $x$ within the compactly supported domain, $u_{i}$ is the function value of $u(\mathrm{x})$ at point $x_{i}, \phi_{i}(\mathrm{x})$ is the weigh function with compact support properties.

Let $J(\mathrm{a}(\mathrm{x}))$ achieve the minimum, then:

$\frac{\partial J}{\partial a(\mathrm{x})}=0$

To get

$\sum_{i=1}^{r} \phi_{i}(\mathrm{x}) p\left(\mathrm{x}_{\mathrm{i}}\right) \mathrm{p}^{\mathrm{T}}\left(\mathrm{x}_{\mathrm{i}}\right) \mathrm{a}(\mathrm{x})=\sum_{i=1}^{r} \phi_{i}(\mathrm{x}) p\left(\mathrm{x}_{\mathrm{i}}\right) u_{i}$

$a(\mathrm{x})=[\mathrm{M}(\mathrm{x})]^{-1} \mathrm{~B}(\mathrm{X}) \mathrm{u}$

Substitute the above expression for Formula (1) to get:

$u^{h}(\mathrm{x})=\mathrm{p}^{\mathrm{T}}(\mathrm{x})[\mathrm{M}(\mathrm{X})]^{-1} \mathrm{~B}(\mathrm{X}) \mathrm{u}$ 
$M(\mathrm{X})=\sum_{i=1}^{r} \phi_{i}(\mathrm{x}) p\left(\mathrm{x}_{\mathrm{i}}\right) \mathrm{p}^{\mathrm{T}}\left(\mathrm{x}_{\mathrm{i}}\right)$

where,

$$
B(\mathrm{X})=\sum_{i=1}^{r} \phi_{i}(\mathrm{x}) p\left(\mathrm{x}_{\mathrm{i}}\right)
$$

Construct the shape function of approximate description $u_{h}(\mathrm{x})=\mathrm{N}_{\mathrm{i}}(\mathrm{X}) \mathrm{u}$ by LSM, then write out the shape function $N_{i}(\mathrm{x})$ at point $x$ according to Formula (6):

$\mathrm{N}_{\mathrm{i}}(\mathrm{x})=\mathrm{p}^{\mathrm{T}}(\mathrm{x})[\mathrm{M}(\mathrm{X})]^{-1} \phi_{i}(\mathrm{X}) \mathrm{p}\left(\mathrm{x}_{\mathrm{i}}\right)$

where, $N_{i}(\mathrm{x})$ is the shape function of partition of unity, $\sum_{i} N_{i}(\mathrm{x})=\phi(\mathrm{x})$ is the extended function.

Extended finite element is based on PUM. In order for any function $\Phi(\mathrm{x})$ to achieve the best approximation, the undermined parameters $q_{i}$ are introduced, then:

$\Phi(\mathrm{x})=\sum_{i} N_{i}(\mathrm{x}) q_{i} \phi(\mathrm{x})$

In XFEM, the unknown field is given more accurate description by adding extenders near the standard field, which are expressed as the following:

$u^{h}(\mathrm{x})=\sum_{j} N_{j}(\mathrm{x}) u_{j}+\Phi(\mathrm{x})$

where, $N_{j}(\mathrm{x})$ is the shape function of standard finite element, $u_{i}$ is the degree of freedom on standard node basis.

$\Phi(\mathrm{x})$ is the extender used to modify the unknown field's properties.

According to Formula (10), the above expression can be rewritten as:

$u^{h}(\mathrm{x})=\sum_{j} N_{j}(\mathrm{x}) u_{j}+\sum_{i} N_{i}(\mathrm{x}) q_{i} \phi(\mathrm{x})$

In Expression (12), the extended function $\phi(x)$ is required to possess some of properties of real solutions of the unknown field $u^{h}(\mathrm{x})$ when being constructed, so as to improve the rate of convergence. In practical application, $\phi(\mathrm{x})$ is typically selected based on the solution space of real solutions, whereas $N_{i}(\mathrm{x})$ generally keeps consistent with $N_{j}(\mathrm{x})$. Provided that $u^{h}(\mathrm{x})$ is a vector field, then degrees of freedom on node basis, $u_{j}$ and $q_{i}$, represent vectors accordingly.

With regard to the crack problem, the above expression can be rewritten as:

$u^{h}(\mathrm{x})=\sum_{i \in I} N_{i}(\mathrm{x}) u_{i}+\sum_{i \in I_{\text {sep }}} N_{i}(\mathrm{x}) \varphi_{\text {step }}(\mathrm{x}) \mathrm{a}_{\mathrm{i}}$

$+\sum_{j=1}^{4} \sum_{i \in I_{i p}} N_{i}(\mathrm{x}) \varphi_{t i p}^{j}(\mathrm{r}, \theta) \mathrm{b}_{i}^{j}$ where, $N_{i}(\mathrm{x})$ is the shape function of standard finite element; $u_{i}$ is the degree of freedom on standard node basis; $a_{i}$ is the degree of freedom on modified node basis related to strong discontinuous function; $b_{i}^{j}$ is the degree of freedom on modified node basis related to elastic asymptotic crack tip function; $I$ is the set of all nodes within the grids; $I_{\text {step }}$ is the set of nodes within the unit incised by the crack; $I_{t i p}$ is the set of nodes within the unit in which the crack tip exists; $\varphi_{\text {step }}(\mathrm{x})$ is a step function, which is used to reflect the displacement jumps at both sides of the crack surface; $\varphi_{t i p}^{j}(\mathrm{r}, \theta)$ is the field function of asymptotic displacement of crack tip, which is used to reflect the singular stress and singular strain of crack tip, and which can be resolved and constructed in light of the crack tip displacement field of planar mixed-mode crack in linear elastic fracture mechanics.

\subsection{Theory of Stress Intensity Factor}

In linear elastic fracture mechanics, the magnitudes of strain field and displacement field at crack tip can be denoted by stress intensity factor. The stress intensity factor is a function about geometrical shape, length of crack and external load, which represents the degree of deformation and load born at crack tip, and which can be used to measure the tendency of or power for crack extension. The methods to compute stress intensity factor mainly include analytical method and numerical method. While the analytical method includes complex function method, weight function method and integral transformation method, the numerical method includes finite element method and more. In extended finite element, mutually interactive integrals are generally adopted to solve the stress intensity factor.

The stress field near crack tip can generally be expressed as:

$$
\left\{\begin{array}{c}
\sigma_{\alpha \beta}=\frac{K_{I}}{\sqrt{2 \pi r}} \sum_{\alpha \beta}^{I}(\theta)+\frac{K_{I I}}{\sqrt{2 \pi r}} \sum_{\alpha \beta}^{I I}(\theta) \\
\sigma_{3 \alpha}=\frac{K_{I I I}}{\sqrt{2 \pi r}} \sum_{3 \alpha}(\theta)
\end{array}\right.
$$

The stress intensity factor may be defined by the stress field near crack tip as:

$$
\left\{\begin{array}{l}
K_{I}=\lim _{r \rightarrow 0} \sqrt{2 \pi r} \sigma_{y}(\mathrm{r}, 0) \\
K_{I I}=\lim _{r \rightarrow 0} \sqrt{2 \pi r} \tau_{x y}(\mathrm{r}, 0) \\
K_{I I I}=\lim _{r \rightarrow 0} \sqrt{2 \pi r} \tau_{y z}(\mathrm{r}, 0)
\end{array}\right.
$$

where $K_{I}$ and $K_{I I}$ may also be defined, using complex function $\Phi(z)$, as:

$$
K=K_{I}-i K_{I I}=2 \sqrt{2 \pi} \lim _{z \rightarrow 0}[\sqrt{z} \Phi(\mathrm{z})]
$$


where, the origin of complex variable $\mathrm{z}$ must be selected at the crack tip.

The dimension of stress intensity factor has such a very particular dimension as [force] [ [length $]^{-3 / 2}$, whose international unit and engineering unit are $N \cdot m^{-3 / 2}$ and $\mathrm{Kg} \cdot \mathrm{mm}^{-3 / 2}$, respectively.

Formula (14) suggests the stress intensity factor is a parameter representing the magnitude of the singular stress field at crack tip, independent of coordinate (x, y). Generally speaking, the magnitude of $\mathrm{K}$ is related to the loading mode, magnitude of load, length and geometrical shape of crack. The status of stress near the crack tip is completely dependent on parameters of stress intensity factor. Once these parameters are decided, The stress field near the crack tip is entirely decided. Thus, for ideal linear elastic materials, Irwin [13] has proposed a norm on fracture of stress intensity factor:

$K_{I}=K_{C}$

Namely, for an I-shape crack, when the $K_{I}$ value achieves the critical $K_{C}$, the crack will start to extend. In the formula $K_{C}$ becomes the toughness of material fracture which is decided by tests and related to test temperature, thickness of board, rate of loading, and environment. Once these external factors are determined, $K_{C}$ becomes a constant independent of loading mode and sample geometry, also of sample size and crack size within a certain range.

Norm (17) on fracture was proposed against I-shape cracks at the very beginning. Under the condition of planar strain, the material of I-shape crack tip is situated in the state of triaxial extension: $\sigma_{y}=\sigma_{x}, \sigma_{z}=v\left(\sigma_{\mathrm{y}}+\sigma_{\mathrm{x}}\right)$. While under the condition of planar stress, the material at the frontier of crack tip is situated in the state of biaxial extension: $\sigma_{y}=\sigma_{x}, \sigma_{z}=0$. Therefore, under the condition of planar strain, the crack is more prone to extending.

\section{STATIC CRACK SIMULATION}

Based on the Abaqus simulation platform, this section is to validate the accuracy of the method in this paper by using XFEM to simulate static cracks and by contrasting the simulation result against the theoretical result.

\subsection{Single-Edge Crack}

As shown in Fig. (1), the length of the board $\mathrm{L}=2 \mathrm{~m}$, the breadth of the board $b=1 \mathrm{~m}$, with a crack on the left side whose length $\mathrm{a}=0.25 \mathrm{~m}$, under the action of tensile stress $\sigma=1 \mathrm{MPa}$, the Poisson ratio $\mu=0.3$ and Young's modulus $\mathrm{E}=2.1 \times 10^{11} \mathrm{~Pa}$. The analytical solution to stress intensity factor is shown as Formula (14) [14].

Fig. (2) is the stress diagram along $\mathrm{x}$ and $\mathrm{y}$ directions, which shows the stress value achieves maximum at the crack tip and diminishes where distant from it. Since the load and the model are longitudinally symmetric along the crack surface, the stress is symmetrically distributed about the crack surface. Computed by Formula (18), the stress intensity factor $\mathrm{K}_{1}=42.04$; computed by finite element software Abaqus, $\mathrm{K}_{1}=41.61$, which is slightly smaller by an error of $\eta=1.0 \%$.

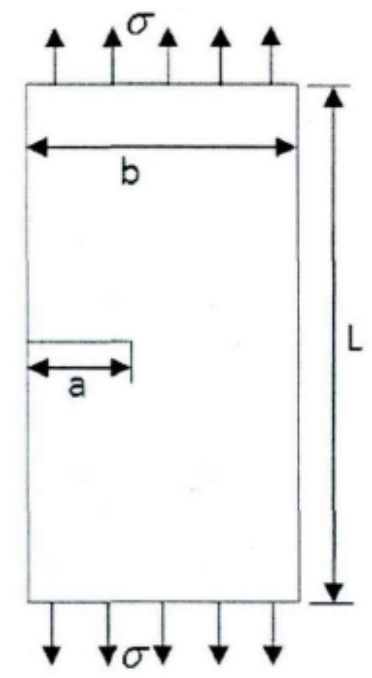

Fig. (1). Single-edge crack.

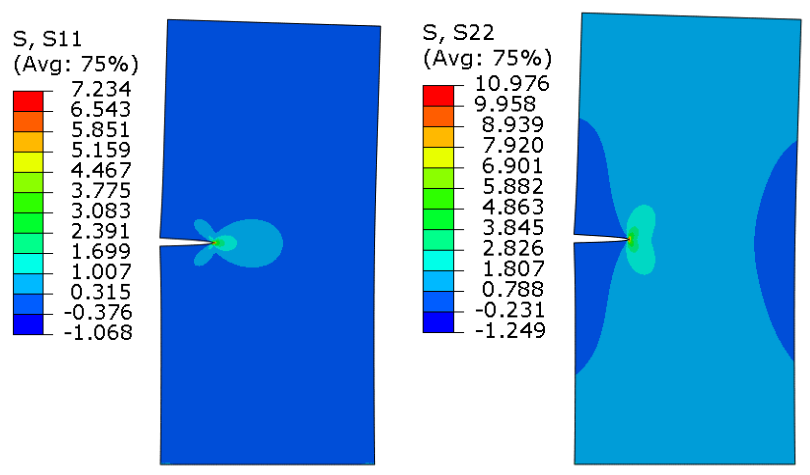

Fig. (2). Stress diagram along $x$ and $y$ directions.

$$
\left\{\begin{array}{c}
K_{1}=\sigma \sqrt{\pi a} F\left(\frac{a}{b}\right) \\
F\left(\frac{a}{b}\right)=\left[\begin{array}{l}
1.12-0.23\left(\frac{a}{b}\right)+10.56\left(\frac{a}{b}\right)^{2} \\
-21.74\left(\frac{a}{b}\right)^{3}+30.42\left(\frac{a}{b}\right)^{4}
\end{array}\right]
\end{array}\right.
$$

\section{2 .Double-Edge Crack}

As shown in Fig. (3), the length of the board $\mathrm{L}=2 \mathrm{~m}$, the breadth of the board $b=1 \mathrm{~m}$, with a crack on both left and right sides whose length $\mathrm{a}=0.25 \mathrm{~m}$, under the action of tensile stress $\sigma=1 \mathrm{MPa}$, the Young's modulus $\mathrm{E}=2.1 \times 10^{11} \mathrm{~Pa}$ and Poisson ratio $\mu=0.3$. The analytical solution to stress intensity factor is shown as Formula (15).

Fig. (4) is the stress diagram along $\mathrm{x}$ and $\mathrm{y}$ directions, which shows the stress value achieves maximum at the crack tip and diminishes where distant from it. Since the load and the model are longitudinally symmetric along the crack surface, the stress is symmetrically distributed about the 
crack surface. Computed by Formula (19), the stress intensity factor $\mathrm{K}_{1}=33.17$; computed by finite element software Abaqus, on the left: $\mathrm{K}_{1}=32.90$, on the right: $\mathrm{K}_{1}=31.99$, which are both slightly smaller by a respective error of $\eta=0.8 \%$ and $\eta=3.6 \%$.

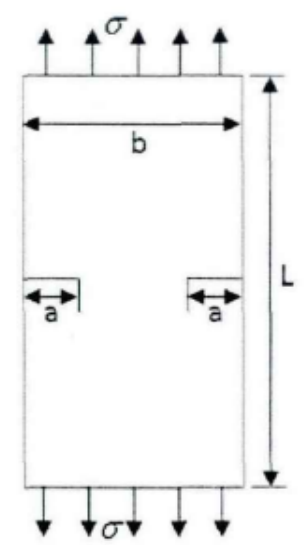

Fig. (3). Double-edge crack.
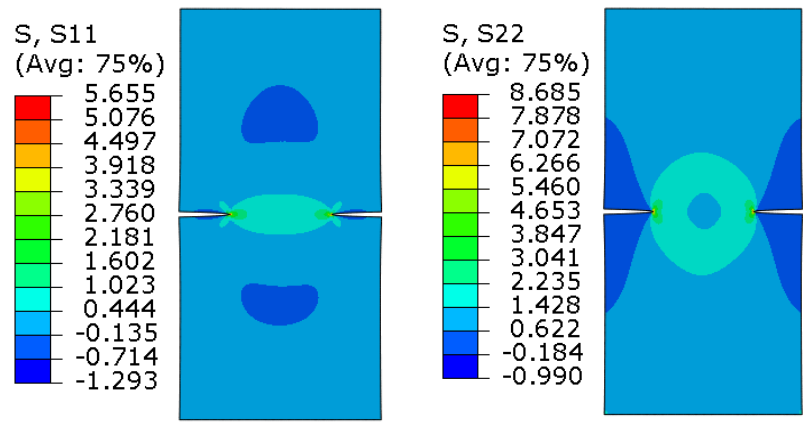

Fig. (4). Stress diagram along $x$ and $y$ directions.

$$
\left\{\begin{array}{c}
K_{1}=\sigma \sqrt{\pi a} g(\xi) \\
\xi=2 a / b \\
g(\xi)=\left(\begin{array}{l}
1.122-0.561 \xi-0.205 \xi^{2} \\
+0.471 \xi^{3}-0.19 \xi^{4}
\end{array}\right) /(1-\xi)^{1 / 2}
\end{array}\right.
$$

\subsection{Central Crack}

As shown in Fig. (5), the length of the board $\mathrm{L}=4 \mathrm{~m}$, the breadth of the board $b=2 \mathrm{~m}$, the length of the central crack $2 \mathrm{a}=0.4 \mathrm{~m}$ which is posed at an angle of $\beta$ between the crack surface and the horizontal axis, under the action of tensile stress $\sigma=1.0 \mathrm{MPa}$, the Young's modulus $E=2.1 \times 10^{11} \mathrm{~Pa}$ and Poisson ratio $\mu=0.3$. The analytical solution to stress intensity factor is shown as Formula (16) [14].

Take $\beta=45^{\circ}$, then the II-type stress intensity factor achieves the maximum, when the I-and II-type stress intensity factors are equal. Through Formula (20), the theoretical solution to stress intensity factor is $K_{I}=K_{I I}=12.5$; through the finite element software, $K_{I}=12.95, K_{I I}=12.80$, the errors being within $4 \%$.

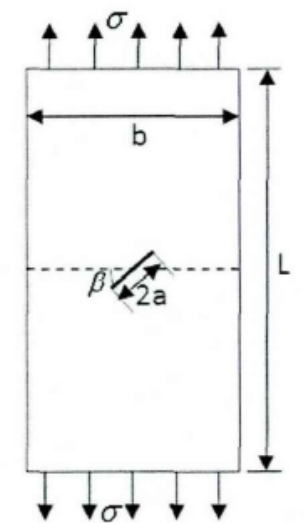

Fig. (5). Central crack.

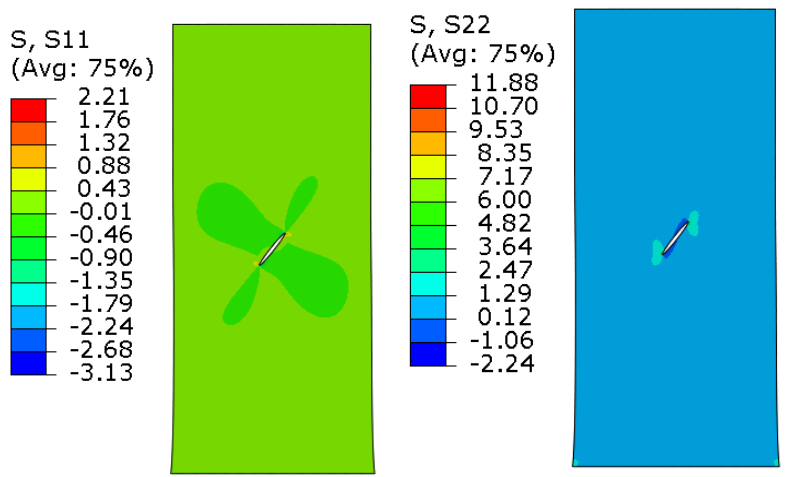

Fig. (6). Stress diagram along $\mathrm{x}$ and $\mathrm{y}$ directions.

$$
\left\{\begin{array}{c}
K_{I}=\sin ^{2}(\beta) \sigma \sqrt{\pi a} \\
K_{I I}=\cos (\beta) \sin (\beta) \sigma \sqrt{\pi a}
\end{array}\right.
$$

Fig. (6) reveals that, the stress is symmetrically distributed about the crack surface, and that the stress value achieves maximum at the crack tip and gradually diminishes where distant from it. The crack surface is a free edge, where the stress value reaches minimum.

\subsection{Two Collinear Cracks}

As shown in Fig. (7), the length of the square board $\mathrm{b}=4 \mathrm{~m}$, the length of the central crack $\mathrm{a}=1 \mathrm{~m}$, the eccentric distance $\mathrm{c}=1 \mathrm{~m}$, under the action of tensile stress $\sigma=1.0 \mathrm{MPa}$, the Young's modulus $E=2.1 \times 10^{11} \mathrm{~Pa}$ and Poisson ratio $\mu=0.3$. The analytical solution to stress intensity factor is shown as Formula (17).

Computed by Formula (21), $K_{A}=45.96, K_{B}=44.58$; computed via the finite element software, $K_{A}=45.80$, $K_{B}=45.92$, both errors being within $3 \%$, which demonstrates the reliability of XFEM and the software. Fig. (8) presents the stress diagram of the collinear cracks along $\mathrm{X}$ and y directions. The stress is symmetrically distributed about the crack surface, the stress value achieves maximum at points $\mathrm{A}$ and $\mathrm{B}$ of the crack tip and gradually diminishes where distant from it. The crack surface is a free edge, where the stress value reaches minimum. 


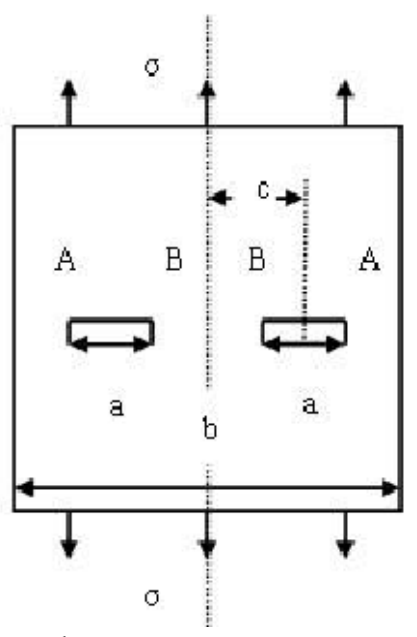

Fig. (7). Co-linear crack.

$$
\left\{\begin{array}{c}
K_{A}=1.16 \sigma \sqrt{\frac{\pi \cdot a}{2}} \\
K_{B}=1.125 \sigma \sqrt{\frac{\pi \cdot a}{2}}
\end{array}\right.
$$
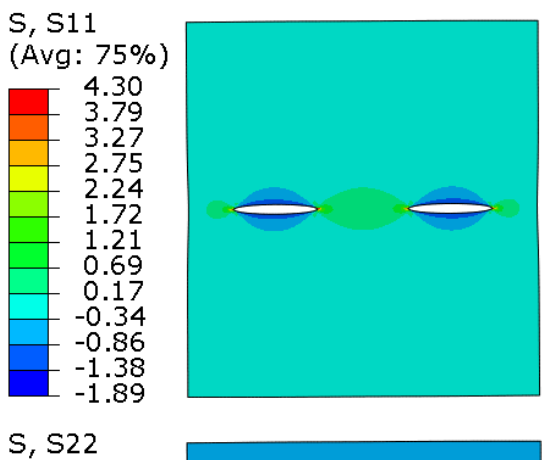

(Avg: 75\%)
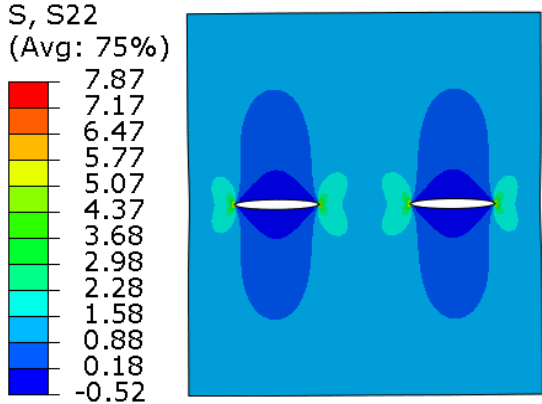

Fig. (8). Stress diagram along $\mathrm{x}$ and $\mathrm{y}$ directions.

\section{CONCLUSION}

This paper first gives a brief introduction to the basic theory on extended finite element, and then uses XFEM for static crack simulation. In the section of static crack simulation, a numerical simulation of static crack has been performed with respect to single-edge crack, double-edge crack, central crack, and collinear cracks, respectively. The reliability of the method in this paper has been validated by comparing the stress intensity factor(s) obtained from simulation with the theoretical stress intensity factor(s).

\section{CONFLICT OF INTEREST}

The authors confirm that this article content has no conflict of interest.

\section{ACKNOWLEDGEMENTS}

Declared none.

\section{REFERENCES}

[1] A. A. Griffith, "The phenomena of rupture and flow in solids," Philosophical Transactions of the Royal Society of London A, vol. 221, pp. 163-198, 1921.

[2] G. R. Irwin, Fracture Dynamics, Fracturing of Metals, American Society of Metals, Cleveland, pp. 147-166, 1948.

[3] G. R. Irwin, "Analysis of stress and strains near the end of a crack transversing a plate," Journal of Applied Mechanics, vol. 24, pp. 109-114, 1957.

[4] J. R. Rice, "A path independent integral and the approximate of strain concentration by notches and cracks," Journal of Applied Mechanics, vol. 35, pp. 379-386, 1968.

[5] J. W. Hutchinson, "Singular behavior at the end of a tensile crack tip in a hardening materials," Journal of Mechanics and Physics of Solids, vol .16, pp. 13-31, 1968.

[6] J. R. Rice, and G. F. Rosengren, "Plane strain deformation near a crack tip in a power law hardening material," Journal of Mechanics and Physics of Solids, vol. 16, pp. 1-12, 1968.

[7] R. M. McMeeting, and D. M. Parks, "Elastic-plastic fracture," American Society for Testing and Materials STP, vol. 668, pp. 175194, 1979.

[8] C.F. Sij, and M. D. German, "Requirement for a one parameter characterization of crack tip fields by the HRR singularity," International Journal of Fracture, vol. 17, pp. 27-43, 1981.

[9] Y. Li, and Z. Wang, "High-order asymptotic solution to i-type nonlinear crack problems of planar strain," Science China A, pp. 182-194, 1986.

[10] T. Belyschko, and T. Black, "Elastic crack growth in finite element with minimal remeshing," International Journal for Numerical Methods in Engineering, vol. 45, no. 5, pp. 601-620, 1999.

[11] N. Moes, J. Dolbow, and T. Belystchko, "A finite element method for crack growth without remeshing," International Journal for Numerical Methods in Engineering, vol. 46, no. 1, pp. 131-150, 1999.

[12] X. Fang, and F. Jin, "ABAQUS platform based extended finite element method," Engineering Mechanics, vol. 24, no.7, pp. 6-10, 2007.

[13] China Aviation Academy, Manual of Stress Intensity Factor, Science Press: Beijing, 1981.

[14] J. Shouyan, and D. Chengbin. "A novel enriched function of elements containing crack tip for fracture analysis in XFEM". Chinese Journal of Theoretical and Applied Mechanics, vol. 45, no. 1, pp.134-138, 2013. 